\title{
The application of service-oriented communication architecture in power grid model service
}

\author{
Pan $\mathrm{Xu}^{1, \text { a }}$, Longda Huang ${ }^{1, \mathrm{~b}}$, Mingyang Sun ${ }^{1, \mathrm{c}}$, Zhang Hong ${ }^{1, \mathrm{~d}}$, Jin Liu ${ }^{1, \mathrm{e}}$ and \\ Yu Fang ${ }^{1, f}$ \\ ${ }^{1}$ China Electric Power Research Institute, Nanjing, 210061, China \\ axupan@epri.sgcc.com.cn, bhuanglongda@epri.sgcc.com.cn, 'sunmingyang@epri.sgcc.com.cn, \\ dzhanghong2@epri.sgcc.com.cn, ${ }^{\mathrm{e}}$ liujin@epri.sgcc.com.cn, ${ }^{\mathrm{f}}$ yufang@epri.sgcc.com.cn
}

Keywords: service-oriented, architecture, power grid communication, model service

Abstract: This paper analyzed the demand and characteristics of power grid communication, stated the features and applications of service-oriented architecture, studied the scenario and method of service-oriented architecture in the power grid communication, proposed a method to organize the power grid communication by service-oriented architecture, and implemented the electric model service by service-oriented architecture, then made a prototype verification in the model service of power grid, in the end, made a conclusion of service-oriented architecture in power grid communication.

\section{Introduction}

\section{Introduction of the communication network of power grid}

Electric power communication network is in order to ensure the security of electric power production and construction, therefore, the business it covered will follow closely to the development of electric power system. Recent years, state grid company focus on the development of UHV transmission technology, the UHV grid line have a long distance and wide range, so it will have a wide range of relay protection and automation data service transmission requirements. The data transfer between the substations which build on the standard IEC61850 rely on the network become an inevitable trend, and the real-time fault data transmission will become an inevitable trend too, with traditional real-time, near real-time and non-real-time business together, will have a combined effect on the construction, development and technical evolution of communication networks.

In recent years, driven by the rapid development of computer technology and information technology, the power system gradually moving towards digitization, information technology and digital technology has become an integral part of power grid system in all aspects. In the architecture of modern power systems, power systems and communications systems have been inseparable, monitoring and control of power systems become more and more dependent on the support of communications systems.

With the development of UHV construction, new energy access and centralized power system progresses, the business of smart grid scheduling control system (D5000) expand rapidly, in the basis of four traditional applications, the monitoring of secondary device, the analysis and evaluation of grid operation, the hybrid simulation decision-making, the pre-assessment of power grid and equipment failure, the power load forecasting and the investment evaluation of distribution network is covered too, so the data size and processing pressure increases rapidly and make the system running in a high pressure, the support and decision-making system becomes more and more complex, the acquisition frequency of grid data from information system will be higher, the scenes for accessing the grid data will become more and more complex. The complexity enhancement means higher management costs and lower reliability, in order to facilitate the data acquisition for power information system, improve the convenience and reliability for communication management; a higher management expectation for the power grid data communication is needed. 


\section{Introduction of the SOA architecture}

Service-oriented architecture (Service-Oriented Architecture, SOA) is not a new software technology, but a new way of software organization and architecture, in this architecture, all modules are packaged into separate services, and provide a separate call interface for these services, by calling these services a certain order to form a business process. Service-oriented architecture is not a kind of software that can be used directly as a technology, but a method to combine the software architecture and business functionality, a method to model, design and deploy discrete logic unit in the software design process. By organize the services into application, there is a lot advantages compared to the call of a single application, the reusability is increased, the development cost of the service is reduced, so the large-scale software pipeline production is possible in such architecture.

SOA is a kind of software architecture with business logic agility and low function coupling feature, the messages transfer between their services is called by the defined service interfaces, you do not need to pay attention to the details of the underlying implementation in order to shield the various enterprise the difference between the software, it has loosely coupled, autonomy and agility features:

The loosely coupled feature is the most influential and most basic features of service-oriented architecture, the services provided by SOA have the feature of low coupling. The low coupling features provided by SOA is mainly in the following aspects: (1) It achieved a macroscopic separation of the service provider and user, so the provider can update the service without the users' concern, in the same time and the user can use the service without the concern of the implementation of the service but only to care the content and interface provided by the service. (2) The implementation of SOA is independent on the special system platform. As SOA only concerned about the interface, the user and provider no need to make any changes with the same interface when the business is changed, so it is independent on system platforms. (3) SOA achieved a low coupling feature in the service of enterprise business. The service in SOA is independent on special business, so it is easy to mix a variety of different services into new services which can be reused by the other service.

The autonomy feature means by the self-organization of the service in SOA, the business logic is encapsulated, so the developers have the greatest degree of independence in terms of logic. In one aspect, the outage technology and the inner business logic is separated by the well defined service of the business logic function, in the other aspect, the autonomy of SOA enhanced the separation of the business logic, application logic and business process logic between the enterprises, so it improved the service logic's auto-encapsulate on the level of the abstraction in the separation of the object environment between the service and the enterprise.

Agility is mainly referred to the changes of the business logic. When the business logic changes, the service will be changed accordingly, in the process of the change, the SOA architecture ensure the developers finished the changes in the lowest cost of time. As the business logic is encapsulated, the outage user only need to communicate with the service as the inner implementation is transparent. So the outage user no need to change the interface and the inner user only need to concern the business logic. Therefore, when there is a change in the enterprise business, the SOA architecture shows great agility in the modeling of the systems.

\section{The business analysis of the model service}

Currently the commonly used interoperability model file for automated scheduling system standard is Q/GDW_216-2008, the data format is suitable for standard IEC61970-555 which is in a high performance format in transform which is based on CIM (Common Information Model). The E language is an efficient power system data markup language based on the object-oriented abstraction of CIM for CIM efficiency defects when described in XML. The E language is a markup language, with the basic features and advantages of markup language; the data instance is a tokenized plain text data. By a few marker symbols and syntax, the E language can describe the power system models in a simple and efficient way, the higher the efficiency of the greater amount of data, and the E language is more in line with the habits of people use than XML, and it is 
convenient for the processing for computer. E language and XML are consistently follow the CIM base object class, the power system model described in E language and XML can be a two-way conversion.

\section{The analysis of model file based on CIM/E}

The E language data is plain text data, the basic content contains commentary, system statement, data block start mark, the data block, the data block end marks and other parts, the class structure is the same to XML. The E language data has two basic structures, the horizontal table structure and the single and multi-column structure, the structure define format is distinguished by the identity of the data block header. In order to ensure the integrity and flexibility, the E language also supports extended syntax.

\section{The preparation of the model data}

This section talks about the preparation of the test data for the communication. According to the requirement of the new generation of the dispatch system model business, this paper made a carding of the power grid model data through the maintain area, the model data is split into three parts, the state model, the province model and the regional model by the principle of regional maintain and universal management. In this paper the state model center is designed to store the state model data, and the province model center is designed to store the province model data and the regional model data.

According to the business of the model maintaining, the state model center and province model center is split into two parts, the model maintain database and the model publish database. The province model maintaining center stores the model information in the maintaining, the province model publish center stores the model information for the running system; the state model maintaining center stores the model information submitted by the province model maintaining center, the state model publish center stores the model information of the running system, it is used for the model's publish.

According to the business processing state, the model data can be split into running state, maintaining state, waiting for confirm, confirm fails and confirm successes. The data in running state is the same to the model of the power grid; it can be used for the construction and calculation in various power grid environments. The data in maintaining state means the data is modified but not confirmed by the superior. The data waiting for confirm means the data is submitted to the superior model center but not confirmed. The data confirm fails means the data submitted is conflict with the exits model data and can't be put into the public center. The data confirm successes means the model data is passed the confirm process and is put into the publish center and can be publish to the production environment.

According to the confirm date, the model data can be marked with a version name generated by the date time, each model version is a backup of the changed model, which can be used for the recall of the model.

Based on the analysis before, the model data center can be architected as bellow: 


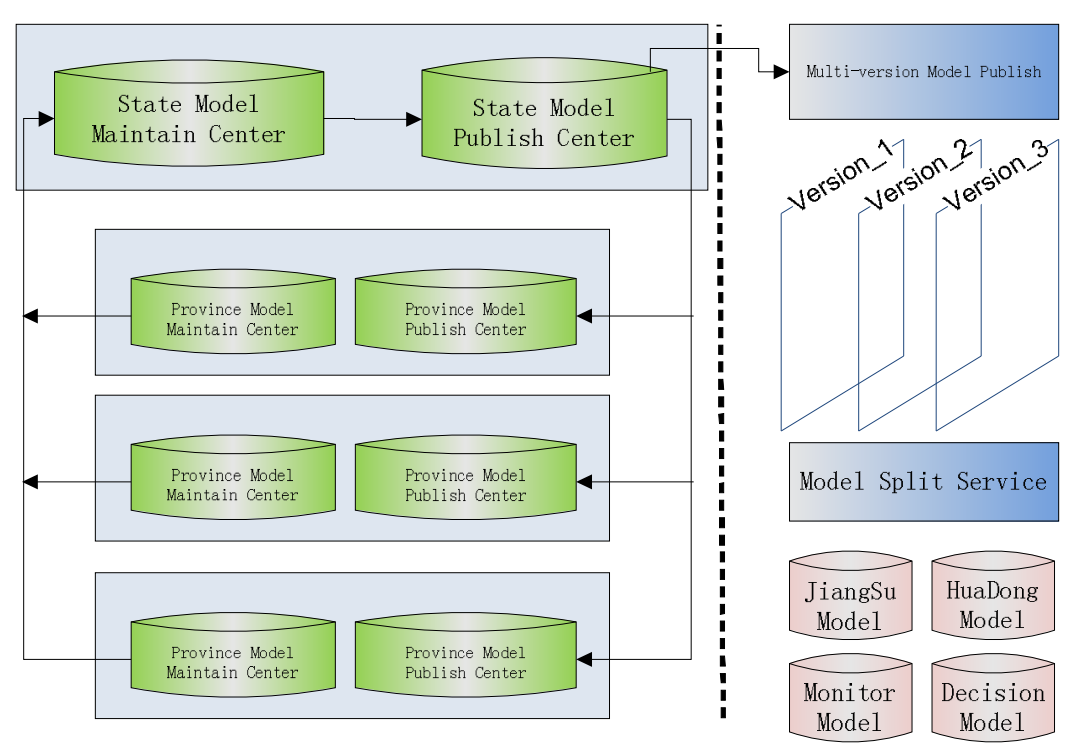

Fig.1 Architecture of the model center

\section{The analysis of the model service}

The create of the service data: the initial province model data comes from three ways, one is put the artificial maintenance model data into the province model data center, the second one is to analyze the existing $\mathrm{CIM} / \mathrm{E}$ file of the substation by automation tools and then put it into the province model data center, the last one is to analyze the existing CIM/E file of the dispatch system and then put it into the province model data center. After the maintenance of the province model data, it will go to the process of confirm, if the data is confirmed successes, then the data will be put into the state model data center.

The analysis of the service in the province model center:

\begin{tabular}{|l|l|}
\hline The service for user login & $\begin{array}{l}\text { Check the login of the province user, if } \\
\text { the user passed the check, return the } \\
\text { corresponding authority. }\end{array}$ \\
\hline The service of model data browse & $\begin{array}{l}\text { Return the province model data based on } \\
\text { the user's input. }\end{array}$ \\
\hline $\begin{array}{l}\text { The service of the model maintenance } \\
\text { and store }\end{array}$ & $\begin{array}{l}\text { After the maintenance of the model data, } \\
\text { store it in the province model center. }\end{array}$ \\
\hline $\begin{array}{l}\text { The service of the static check of the } \\
\text { model data }\end{array}$ & $\begin{array}{l}\text { Check the range of the model data based } \\
\text { on the existing model data in the province } \\
\text { model maintain center. }\end{array}$ \\
\hline $\begin{array}{l}\text { The service of the model submit and } \\
\text { check }\end{array}$ & $\begin{array}{l}\text { If the model maintained passed the static } \\
\text { check, then store it into the state model } \\
\text { maintain center. }\end{array}$ \\
\hline
\end{tabular}

Table 1 
The analysis of the service in the state model center:

\begin{tabular}{|l|l|}
\hline The service for user login & $\begin{array}{l}\text { Check the login of the state user, if the } \\
\text { user passed the check, return the } \\
\text { corresponding authority. }\end{array}$ \\
\hline The service of model query & $\begin{array}{l}\text { Return the state model data based on the } \\
\text { user's input. }\end{array}$ \\
\hline The service of dynamic check & $\begin{array}{l}\text { Check boundary of the submitted } \\
\text { province model data based on the state } \\
\text { model data. }\end{array}$ \\
\hline $\begin{array}{l}\text { The service of the browse of the model } \\
\text { data waiting for confirm }\end{array}$ & $\begin{array}{l}\text { Provide a service to browse the model } \\
\text { data waiting for confirm from the } \\
\text { province center. }\end{array}$ \\
\hline The service of the confirm result & $\begin{array}{l}\text { Provide a service for the province user to } \\
\text { get the confirm result. }\end{array}$ \\
\hline $\begin{array}{l}\text { The service of the model data in specific } \\
\text { version }\end{array}$ & $\begin{array}{l}\text { Provide a service to browse the whole } \\
\text { model data or a partial model data in a } \\
\text { specific time range. }\end{array}$ \\
\hline The service of the model publish & $\begin{array}{l}\text { Publish the model data which is } \\
\text { confirmed successes to the province } \\
\text { model center. }\end{array}$ \\
\hline
\end{tabular}

Table 2

The schematic diagram of the model service is in the below:

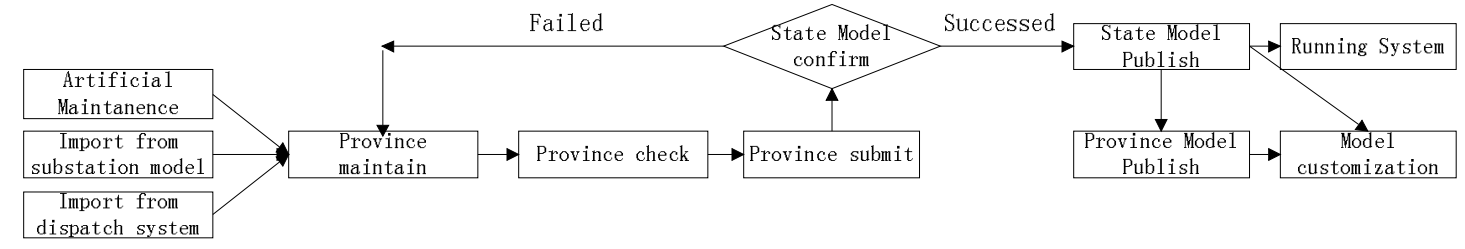

Fig.2 Model service process follow diagram

\section{Service Architecture}

\section{The main architecture}

After the analysis of the model service process, the architecture diagram of the service-oriented model service is in the following: at the beginning of the architecture, the main class of the model service is extracted out, then the service function in the main part is analyzed, after that the main class is will be registered into the service broker application, after the register, the user can send the service request to the service broker application, the broker will redirect the request to the specific service, after the return the service request is finished. 


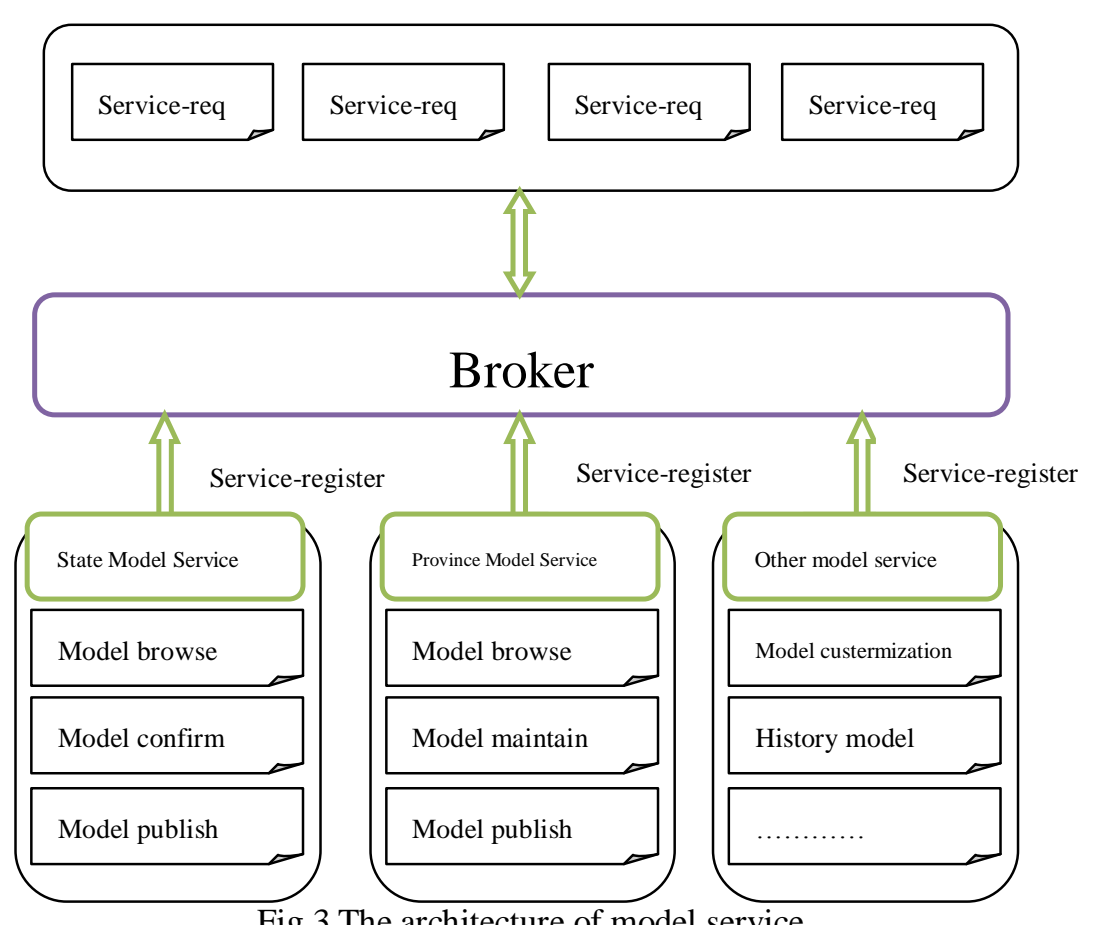

Fig. 3 The architecture of model service

The broker works as a service agency, it has the following functions:

I The service register, after the register of the service, it will store the ip address and port of the service, then store it as a service description file locally. The file will be used to construct the service pool when broker downs or restarted. The service worker need to provide the ip address, port, the service description, the parameters and type for calling the service in the process of service register. When the broker accepted the register request, it will store all the information to the local service description file, and return a unique service identity to the service worker.

I The service remove, the service worker send a service delete request to the broker, when the broker accepted the request, it will mark the corresponding service as unusable and then delete the relater information of the specific service in the description file.

I The service request and reply, the service user send a service request to the broker, with the corresponding service parameter, the broker will locate the service and redirect the request to the service worker, and then transmit the result to the service user.

\section{The protocol of communication}

Based on the model service architecture, there are three main service classes, they are the state model service, the province model service and the other model service, there are several service functions in the main service class. In each service request, the service user need to send the service identity, the service function name and the service request parameter to the service worker, so the service agreement can be divided into two parts, the first part is the location service, include the service identity and the service function name, the second part is the service request parameters, the structure of the service protocol is showing below:

\begin{tabular}{|l|l|l|l|}
\hline Service identity & Service function name & parameter number & parameter buff \\
\hline Int & char[32] & Int & StrPara* \\
\hline
\end{tabular}

Table 3

The result of the service request includes two parts. The first part is the description of the request result, if the request is successes, then the second part will be the reply data of the request, include the start address and length of the data, the structure of the service reply protocol is showing below:

\begin{tabular}{|l|l|l|l|}
\hline Service identity & service result description & start address & data length \\
\hline Int & char[64] & char* & Int \\
\hline
\end{tabular}

Table 4 


\section{Conclusion}

This paper firstly analyzed the current communication status, the main business and technical requirement of power grid, secondly studied the theory and characteristic of SOA, and make a research on the business of power model communication, then make a SOA modeling on model data and model business, at the end, make a architecture and design of the model service based on SOA.

\section{References}

[1] Xing Yaozhong. A method to share model information of the power dispatch center based on CIM/E. Power grid automation Vol.37 No.8 Apr.25,2013.

[2] Ma Taotao, Guo Chuangxin, Cao Yijia, et al. Current status and development trend of intelligent dispatching automation system of power system[J]. Automation of Electric Power Systems. 2010,34(9)7-9.

[3] Xie Dawei, Yang Xiaozhong. Implementation of networking telecontrol technique in dispatching automation system[J]. Power System Technology,2004,28(8) 34-37(in Chinese).

[4] IEC 61970-555: CIM Based Efficient Model Exchange Format (CIM/E) [S].2012.

[5] Q/GDW_215-2008: Data Markup Language for Electric Power System -E Language.

[6] Service Oriented Architecture(SOA) Security Models. [D]. Engineering, Computer 2011 CNKI 